\title{
Bilateral comparison on transmission coefficient measurements between TÜBITAK UME and SASO NMCC
}

\author{
Handan Sakarya ${ }^{1, *}$, Murat Celep ${ }^{1}$, Ahmed Hamad AlJawan ${ }^{2}$, Ibrahim Mohammed $^{2}$ \\ AlBoraih $^{2}$ and Khalid AlDawood ${ }^{2}$ \\ ${ }^{1}$ National Metrology Institute (TUBITAK UME), Baris Mah Dr. Zeki Acar Cad. No.1 Gebze-Kocaeli, \\ Turkey \\ ${ }^{2}$ National Measurement and Calibration Center (NMCC), Saudi Standards, Metrology and Quality \\ Organization (SASO), Al Muhammadiyah-in front of King Saud University PO. B 343711471 \\ Riyadh, Kingdom of Saudi Arabia
}

\begin{abstract}
This paper describes a bilateral comparison between two national metrology institutes, National Metrology Institute of Turkey (TÜBİTAK UME) and National Measurement and Calibration Center at Saudi Standards, Metrology and Quality Organization of the Kingdom of Saudi Arabia (SASO NMCC). The aim of the comparison is to check the newly installed SASO NMCC radio frequency and microwave laboratory infrastructure and assess compatibility between two institutes. Measured quantity of the comparison is transmission coefficient of fixed attenuators. The comparison was carried out in three months with $3 \mathrm{~dB}, 6 \mathrm{~dB}, 10 \mathrm{~dB}$, $20 \mathrm{~dB}$ and $30 \mathrm{~dB}$ fixed attenuators. Measurements were performed using commercial Vector Network Analyzer in controlled environmental conditions. Two laboratories were used same measurement technique called as short, open, load and through. The analysis of the results provides good agreement between TÜBITTAK UME and SASO NMCC as a conclusion.
\end{abstract}

\section{Introduction}

National Measurement and Calibration Center of the Kingdom of Saudi Arabia (SASO NMCC) newly installed radio frequency (RF) and microwave laboratory. The laboratory frequency range is between $100 \mathrm{kHz}$ to $50 \mathrm{GHz}$ with type $\mathrm{N}, 3.5 \mathrm{~mm}$ and $2.4 \mathrm{~mm}$ connector types for scattering (S) parameters measurement. S-parameters are transmission coefficient and reflection coefficient with measurement range from $0 \mathrm{~dB}$ to $-60 \mathrm{~dB}$ and from -1 to +1 respectively. In order to check S-parameters measurement infrastructure and establish international coherence of SASO NMCC, the National Metrology Institute of Turkey (TÜBİTAK UME) and SASO NMCC have organized and carried out a bilateral comparison on transmission coefficient measurement in 2016. Additionally, it is asked to measure reflection coefficient of the travelling standards. TÜBİTAK UME was acting as a

\footnotetext{
*Corresponding author: handan.sakarya@tubitak.gov.tr
} 
pilot laboratory for the bilateral comparison and provided a technical protocol to define the measurement and organisational details according to BIPM guideline [1]. TÜBİTAK UME provided five travelling standards have type $\mathrm{N}$ connectors and traceable to the International System of Units (SI). The comparison was carried out in three months with $3 \mathrm{~dB}, 6 \mathrm{~dB}, 10$ $\mathrm{dB}, 20 \mathrm{~dB}$ and $30 \mathrm{~dB}$ fixed attenuators. In this paper, results of this comparison exercise using attenuators are described.

\section{Travelling standards and time schedule}

Travelling standards belonging to the TÜBİTAK UME and identification of each fixed attenuator are described in Table 1. These standards were chosen for their high accuracy and stability in time.

Table 1. Travelling standards.

\begin{tabular}{|c|c|c|c|c|}
\hline Device & $\begin{array}{l}\text { Nominal } \\
\text { value }\end{array}$ & Type & Serial number & Connector \\
\hline \multirow{5}{*}{$\begin{array}{l}\text { Fixed } \\
\text { attenuator }\end{array}$} & $3 \mathrm{~dB}$ & \multirow{5}{*}{$\begin{array}{c}\text { Agilent } \\
8491 \mathrm{~B}\end{array}$} & MY39265166 & \multirow{5}{*}{ Male/Female } \\
\hline & $6 \mathrm{~dB}$ & & MY39265283 & \\
\hline & $10 \mathrm{~dB}$ & & MY39265254 & \\
\hline & $20 \mathrm{~dB}$ & & MY39265284 & \\
\hline & $30 \mathrm{~dB}$ & & MY39263978 & \\
\hline
\end{tabular}

Circulation of the travelling standards was organized as 2 weeks to carry out measurements and 2 weeks for transportation. Travelling standards were measured totally two times by TÜBİTAK UME before (UME1) and after (UME2) the measurement of SASO NMCC.

\section{S-Parameter measurement method and results}

S-parameter measurements were made using commercial vector network analyzers (VNA) belonging to the TÜBİTAK UME (Rohde\&Schwarz ZVA50) and SASO NMCC (Keysight $\mathrm{N} 5225 \mathrm{~A}$ ) in controlled environmental conditions. VNA calibration was carried out using Agilent 85054B type $\mathrm{N}$ calibration kit with short-open-load-through (SOLT) calibration method at both of the NMIs. Male and female end of the travelling standards were connected to port 1 and port 2 of the VNA respectively for all measurements reported here.

Results were delivered to the pilot laboratory in the format of real, imaginary and logarithmic $(\mathrm{dB})$ value. The uncertainties of measurement were calculated according to JCGM 100 "Guide to the expression of uncertainty measurement" and "EA-4/02 expression of the uncertainty of measurement calibration" documents for the coverage probability of approximately $95 \%[2,3]$. Since VNA was used in the measurements, the uncertainty was calculated in accordance with the EURAMET guidelines for VNA [4]. The uncertainty in S-parameter measurements was evaluated by using excel macro software developed by TÜBITAK UME which is including uncertainty components, i.e. repeatability, reproducibility, VNA non-linearity, mismatch, cable stability etc.

Approved measurement frequencies and attenuation values for the comparison are $50 \mathrm{MHz}$, $100 \mathrm{MHz}, 300 \mathrm{MHz}, 500 \mathrm{MHz}, 1 \mathrm{GHz}$ to $18 \mathrm{GHz}$ with $1 \mathrm{GHz}$ step and $3 \mathrm{~dB}, 6 \mathrm{~dB}, 10 \mathrm{~dB}$, $20 \mathrm{~dB}, 30 \mathrm{~dB}, 40 \mathrm{~dB}, 50 \mathrm{~dB}, 60 \mathrm{~dB}$ respectively. Although, attenuation values at aforementioned frequencies were measured and reported to the pilot laboratory, the uncertainty budget declared only for $50 \mathrm{MHz}, 1 \mathrm{GHz}, 10 \mathrm{GHz}$ and $18 \mathrm{GHz}$ frequency point measurements. Also reflection coefficient measurement results of the $40 \mathrm{~dB}, 50 \mathrm{~dB}$ and 60 
$\mathrm{dB}$ attenuators were not declared because the connection order of the attenuators was not defined in the protocol.

Comparison reference values " $\mathrm{X}_{r e f}$ " (Eq. 1) and associated uncertainties "U $\mathrm{U}_{\text {ref }}$ " (Eq. 2) were calculated using the measurement results of TÜBITTAK UME.

$$
\begin{aligned}
& x_{r e f}=\frac{U M E 1+U M E 2}{2} \\
& U_{\text {ref }}=\frac{1}{2} \sqrt{U_{U M E 1^{2}+U_{U M E 2}{ }^{2}}}
\end{aligned}
$$

Measurement results of the laboratories and comparison reference value with expanded uncertainties were given in Table 2.

Table 2. Measurement results of the laboratories and comparison reference value with expanded

\begin{tabular}{|c|c|c|c|c|c|c|c|c|c|}
\hline \multirow{2}{*}{ Attn } & \multirow{2}{*}{$\begin{array}{l}\text { Freq. } \\
\text { (GHz) }\end{array}$} & \multicolumn{2}{|c|}{ UME1 } & \multicolumn{2}{|c|}{ SASO NMCC } & \multicolumn{2}{|c|}{ UME2 } & \multicolumn{2}{|c|}{ Reference Value } \\
\hline & & $\left|\mathrm{S}_{21}\right|(\mathrm{dB})$ & $\mathrm{U}(\mathrm{dB})$ & $\left|\mathrm{S}_{21}\right|(\mathrm{dB})$ & $\mathrm{U}(\mathrm{dB})$ & $\mid \mathrm{S}_{2 \mathrm{~J}} \mathrm{l}(\mathrm{dB})$ & $\mathrm{U}(\mathrm{dB})$ & $\left|\mathrm{S}_{21}\right|(\mathrm{dB})$ & $\mathrm{U}(\mathrm{dB})$ \\
\hline \multirow{4}{*}{$3 \mathrm{~dB}$} & 0.05 & 3.076 & 0.012 & 3.074 & 0.012 & 3.078 & 0.012 & 3.077 & 0.008 \\
\hline & 1 & 3.097 & 0.012 & 3.100 & 0.012 & 3.098 & 0.012 & 3.098 & 0.008 \\
\hline & 10 & 2.782 & 0.014 & 2.791 & 0.012 & 2.776 & 0.012 & 2.779 & 0.009 \\
\hline & 18 & 2.817 & 0.033 & 2.817 & 0.032 & 2.840 & 0.032 & 2.829 & 0.023 \\
\hline \multirow{4}{*}{$6 \mathrm{~dB}$} & 0.05 & 6.013 & 0.015 & 6.010 & 0.030 & 6.011 & 0.015 & 6.012 & 0.011 \\
\hline & 1 & 6.045 & 0.015 & 6.048 & 0.030 & 6.042 & 0.015 & 6.044 & 0.011 \\
\hline & 10 & 6.090 & 0.015 & 6.093 & 0.030 & 6.087 & 0.015 & 6.089 & 0.011 \\
\hline & 18 & 5.848 & 0.021 & 5.855 & 0.031 & 5.843 & 0.021 & 5.846 & 0.015 \\
\hline \multirow{4}{*}{$10 \mathrm{~dB}$} & 0.05 & 10.297 & 0.020 & 10.296 & 0.049 & 10.302 & 0.020 & 10.299 & 0.014 \\
\hline & 1 & 10.341 & 0.021 & 10.347 & 0.049 & 10.345 & 0.021 & 10.343 & 0.015 \\
\hline & 10 & 10.420 & 0.021 & 10.426 & 0.049 & 10.426 & 0.021 & 10.423 & 0.015 \\
\hline & 18 & 10.427 & 0.032 & 10.408 & 0.050 & 10.414 & 0.032 & 10.420 & 0.023 \\
\hline \multirow{4}{*}{$20 \mathrm{~dB}$} & 0.05 & 19.402 & 0.032 & 19.363 & 0.036 & 19.261 & 0.036 & 19.331 & 0.025 \\
\hline & 1 & 19.749 & 0.036 & 19.809 & 0.036 & 19.768 & 0.036 & 19.758 & 0.025 \\
\hline & 10 & 20.077 & 0.036 & 20.084 & 0.036 & 20.058 & 0.036 & 20.067 & 0.025 \\
\hline & 18 & 20.097 & 0.036 & 20.095 & 0.036 & 20.104 & 0.036 & 20.101 & 0.025 \\
\hline \multirow{4}{*}{$30 \mathrm{~dB}$} & 0.05 & 30.462 & 0.055 & 30.454 & 0.143 & 30.464 & 0.055 & 30.463 & 0.039 \\
\hline & 1 & 30.485 & 0.055 & 30.494 & 0.141 & 30.487 & 0.055 & 30.486 & 0.039 \\
\hline & 10 & 30.221 & 0.054 & 30.229 & 0.139 & 30.226 & 0.054 & 30.223 & 0.038 \\
\hline & 18 & 30.758 & 0.057 & 30.766 & 0.142 & 30.765 & 0.057 & 30.761 & 0.040 \\
\hline \multirow{4}{*}{$40 \mathrm{~dB}$} & 0.05 & 40.753 & 0.075 & 40.752 & 0.200 & 40.763 & 0.075 & 40.758 & 0.053 \\
\hline & 1 & 40.830 & 0.076 & 40.842 & 0.188 & 40.835 & 0.076 & 40.833 & 0.053 \\
\hline & 10 & 40.653 & 0.074 & 40.654 & 0.187 & 40.647 & 0.074 & 40.650 & 0.052 \\
\hline & 18 & 41.195 & 0.074 & 41.226 & 0.187 & 41.193 & 0.074 & 41.194 & 0.052 \\
\hline \multirow{4}{*}{$50 \mathrm{~dB}$} & 0.05 & 49.950 & 0.087 & 49.862 & 0.192 & 50.094 & 0.089 & 50.022 & 0.062 \\
\hline & 1 & 50.139 & 0.087 & 50.099 & 0.090 & 50.167 & 0.087 & 50.153 & 0.062 \\
\hline & 10 & 50.333 & 0.087 & 50.323 & 0.087 & 50.327 & 0.087 & 50.330 & 0.062 \\
\hline & 18 & 50.858 & 0.091 & 50.886 & 0.091 & 50.866 & 0.091 & 50.862 & 0.064 \\
\hline \multirow{4}{*}{$60 \mathrm{~dB}$} & 0.05 & 59.85 & 0.26 & 60.42 & 0.45 & 60.34 & 0.18 & 60.09 & 0.18 \\
\hline & 1 & 60.54 & 0.11 & 60.53 & 0.28 & 60.52 & 0.11 & 60.53 & 0.08 \\
\hline & 10 & 60.76 & 0.11 & 60.79 & 0.28 & 60.76 & 0.11 & 60.76 & 0.08 \\
\hline & 18 & 61.23 & 0.12 & 61.24 & 0.29 & 61.29 & 0.12 & 61.26 & 0.09 \\
\hline
\end{tabular}
uncertainties.

Degrees of equivalence, DoE, is calculated by subtracting the reference value from the each measurements (Eq. 3) and its uncertainty is calculated according to the Equation 4.

$$
\begin{gathered}
D o E=x_{\text {lab }}-x_{r e f} \\
U_{D o E}=\sqrt{{U_{r e f}{ }^{2}+U_{l a b}{ }^{2}}^{2}}
\end{gathered}
$$

where, 
$x_{l a b}$ : Participant laboratory measurement result

$U_{l a b}$ : Participant laboratory measurement uncertainty

Degrees of equivalence with respect to the reference value of each measurement can be found in Table 3.

Table 3. DoE with respect to the reference value.

\begin{tabular}{|c|c|c|c|c|c|c|c|}
\hline \multirow{2}{*}{ Attn } & \multirow{2}{*}{$\begin{array}{l}\text { Freq. } \\
\text { (GHz) }\end{array}$} & \multicolumn{2}{|c|}{ UME1 } & \multicolumn{2}{|c|}{ SASO NMCC } & \multicolumn{2}{|c|}{ UME2 } \\
\hline & & $\mathrm{DoE}(\mathrm{dB})$ & $\mathrm{U}_{\mathrm{DoE}}(\mathrm{dB})$ & DoE (dB) & $\mathrm{U}_{\mathrm{DoE}}(\mathrm{dB})$ & $\operatorname{DoE}(\mathrm{dB})$ & $\mathrm{U}_{\mathrm{DoE}}(\mathrm{dB})$ \\
\hline \multirow{4}{*}{$3 \mathrm{~dB}$} & 0.05 & 0.001 & 0.015 & 0.003 & 0.015 & -0.001 & 0.015 \\
\hline & 1 & 0.001 & 0.015 & -0.003 & 0.015 & -0.001 & 0.015 \\
\hline & 10 & -0.003 & 0.016 & -0.012 & 0.015 & 0.003 & 0.015 \\
\hline & 18 & 0.012 & 0.040 & 0.012 & 0.040 & -0.012 & 0.039 \\
\hline \multirow{4}{*}{$6 \mathrm{~dB}$} & 0.05 & -0.001 & 0.018 & 0.002 & 0.031 & 0.001 & 0.018 \\
\hline & 1 & -0.001 & 0.018 & -0.005 & 0.031 & 0.001 & 0.018 \\
\hline & 10 & -0.002 & 0.018 & -0.004 & 0.032 & 0.002 & 0.018 \\
\hline & 18 & -0.003 & 0.026 & -0.010 & 0.034 & 0.003 & 0.026 \\
\hline \multirow{4}{*}{$10 \mathrm{~dB}$} & 0.05 & 0.002 & 0.025 & 0.003 & 0.051 & -0.002 & 0.025 \\
\hline & 1 & 0.002 & 0.025 & -0.004 & 0.051 & -0.002 & 0.025 \\
\hline & 10 & 0.003 & 0.025 & -0.003 & 0.051 & -0.003 & 0.025 \\
\hline & 18 & -0.006 & 0.039 & 0.012 & 0.055 & 0.006 & 0.039 \\
\hline \multirow{4}{*}{$20 \mathrm{~dB}$} & 0.05 & -0.071 & 0.109 & -0.032 & 0.109 & 0.071 & 0.109 \\
\hline & 1 & 0.010 & 0.046 & -0.050 & 0.046 & -0.010 & 0.046 \\
\hline & 10 & -0.009 & 0.044 & -0.017 & 0.044 & 0.009 & 0.044 \\
\hline & 18 & 0.003 & 0.044 & 0.005 & 0.044 & -0.003 & 0.044 \\
\hline \multirow{4}{*}{$30 \mathrm{~dB}$} & 0.05 & 0.001 & 0.067 & 0.009 & 0.148 & -0.001 & 0.067 \\
\hline & 1 & 0.001 & 0.068 & -0.008 & 0.146 & -0.001 & 0.068 \\
\hline & 10 & 0.003 & 0.066 & -0.005 & 0.145 & -0.003 & 0.066 \\
\hline & 18 & 0.004 & 0.070 & -0.005 & 0.147 & -0.004 & 0.070 \\
\hline \multirow{4}{*}{$40 \mathrm{~dB}$} & 0.05 & 0.005 & 0.092 & 0.006 & 0.207 & -0.005 & 0.092 \\
\hline & 1 & 0.002 & 0.092 & -0.009 & 0.196 & -0.002 & 0.093 \\
\hline & 10 & -0.003 & 0.091 & -0.004 & 0.194 & 0.003 & 0.091 \\
\hline & 18 & -0.001 & 0.091 & -0.032 & 0.194 & 0.001 & 0.091 \\
\hline \multirow{4}{*}{$50 \mathrm{~dB}$} & 0.05 & 0.072 & 0.107 & 0.160 & 0.202 & -0.072 & 0.109 \\
\hline & 1 & 0.014 & 0.107 & 0.054 & 0.109 & -0.014 & 0.107 \\
\hline & 10 & -0.003 & 0.107 & 0.007 & 0.107 & 0.003 & 0.107 \\
\hline & 18 & 0.004 & 0.111 & -0.024 & 0.112 & -0.004 & 0.111 \\
\hline \multirow{4}{*}{$60 \mathrm{~dB}$} & 0.05 & 0.245 & 0.314 & -0.332 & 0.481 & -0.245 & 0.318 \\
\hline & 1 & -0.010 & 0.136 & 0.001 & 0.291 & 0.010 & 0.136 \\
\hline & 10 & 0.001 & 0.139 & -0.035 & 0.292 & -0.001 & 0.139 \\
\hline & 18 & 0.030 & 0.147 & 0.021 & 0.298 & -0.030 & 0.148 \\
\hline
\end{tabular}

DoE for $1 \mathrm{GHz}$ and $10 \mathrm{GHz}$ of $10 \mathrm{~dB}$ and $60 \mathrm{~dB}$ fixed attenuators respectively are given in Figure 1.

$10 \mathrm{~dB} @ 1 \mathrm{GHz}$

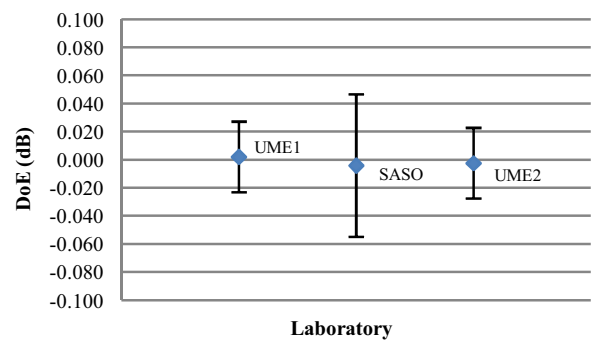

$60 \mathrm{~dB} @ 18 \mathrm{GHz}$

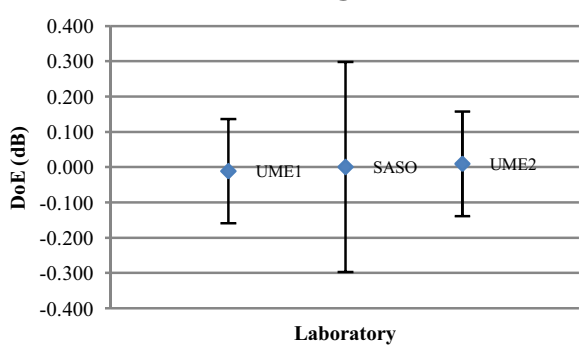

Fig. 1. DoE of $10 \mathrm{~dB} @ 1 \mathrm{GHz}$ and $60 \mathrm{~dB} @ 10 \mathrm{GHz}$. 


\section{Conclusion}

The results from a bilateral measurement comparison of fixed attenuators' transmission coefficient, performed between TÜBİTAK UME and SASO NMCC, are reported. . The comparison involved two port devices have type $\mathrm{N}$ connector as travelling standards and completed within three months. All the results are presented at Table 2 and Table 3 with corresponding uncertainties are compatible within the stated uncertainties. As stated in Table 3, while calculated maximum DoE value is lower than $\pm 0.075 \mathrm{~dB}$ up to $50 \mathrm{~dB}$ fixed attenuator, this value is increasing $\pm 0.34 \mathrm{~dB}$ for $60 \mathrm{~dB}$ fixed attenuator. Finally, the comparison is regarded as successfully and the newly installed SASO NMCC radio frequency and microwave laboratory S-parameters measurement infrastructure is sufficient and measurement results of two institutes are compatible.

\section{References}

1. CCEM Guidelines for Planning, Organizing, Conducting and Reporting Key, Supplementary and Pilot Comparisons (available on the BIPM website: http://www.bipm.org/utils/common/pdf/CC/CCEM/ccem_guidelines.pdf) (2007)

2. Evaluation of measurement data - Guide to the Expression of Uncertainty in Measurement (GUM), JCGM 100, First edition (available on the BIPM website: http://www.bipm.org/utils/common/documents/jcgm/JCGM_100_2008_E.pdf) (2008)

3. EA 4/02, "Evaluation of the uncertainty of measurement in calibration", Rev 01 (2013)

4. EURAMET cg-12, "Guidelines on the evaluation of vector network analyzers (VNA)", Ver. $2.0(2011)$ 